Mathematical Modelling and AnAlysis

Volume 22 Number 4, July 2017, 484-502

https://doi.org/10.3846/13926292.2017.1329171

(c) Vilnius Gediminas Technical University, 2017
Publisher: Taylor\&Francis and VGTU

http://www.tandfonline.com/TMMA

ISSN: $1392-6292$

eISSN: $1648-3510$

\title{
Global Exponential Stability of Almost Periodic Solutions for Nicholson's Blowflies System with Nonlinear Density-Dependent Mortality Terms and Patch Structure
}

\author{
Pengyan Liu, Liang Zhang, Shitao Liu and Lifei Zheng \\ College of Science, Northwest A $B F$ University \\ No. 22 Xinong Road, Yangling, 712100 Shaanxi, China \\ E-mail(corresp.): zhanglsd@126.com
}

Received February 26, 2017; revised May 5, 2017; published online July 15, 2017

\begin{abstract}
This paper considers a generalized Nicholson's blowflies system with nonlinear density-dependent mortality terms and patch structure. Under appropriate conditions, we establish some criteria to ensure that the solutions of this system exist and converge globally exponentially to a positive almost periodic solution. The results complement another case of nonlinear density-dependent mortality terms in Chen and Wang [5].
\end{abstract}

Keywords: nonlinear density-dependent mortality term, patch structure, Nicholson's blowflies system, global exponential stability, almost periodic solution.

AMS Subject Classification: 92D25; 34D23.

\section{Introduction}

To describe the population of the Australian sheep blowfly and agree with the experimental date of Nicholson [20], Gurney et al. [9] proposed the following Nicholson's blowflies equation

$$
N^{\prime}(t)=-\delta N(t)+p N(t-\tau) e^{-a N(t-\tau)} .
$$

Here, $N(t)$ is the size of the population at time $t, p$ is the maximum per capita daily egg production, $\frac{1}{a}$ is the size at which the population reproduces at its maximum rate, $\delta$ is the per capita daily adult death rate, and $\tau$ is the generation time.

As we all know, the study of stability for the nonlinear models (see e.g. $[1,6,19,24,26,27,32,33])$ not only has profound practical significance, but also will enrich and perfect the theory of nonlinear equations to some extent. In the past forty years, the theory of the Nicholson's blowflies equation has made a remarkable progress with main results scattered in numerous research 
papers. In particular, there have been extensive studies on the problem of the existence of positive periodic solutions for the classical Nicholson's model and some generalizations with variable coefficients and delays. We refer the reader to $[14,16,18,21]$ and the references cited therein.

In 2010, Berezansky et al. [2] pointed out that a new study indicates that a linear model of density-dependent mortality will be most accurate for populations at low densities. And there have been extensive results on the problem of the existence of positive almost periodic solutions for Nicholson's blowflies equation without nonlinear density-dependent mortality term in the literature $[4,13,17,30]$. Berezansky et al. [2] presented the following Nicholson's blowflies model with a nonlinear density-dependent mortality term

$$
N^{\prime}(t)=-D(N(t))+P N(t-\tau) e^{-a N(t-\tau)},
$$

where $P$ is a positive constant and function $D$ might have one of the following forms: $D(N)=\frac{a N}{N+b}$ or $D(N)=a-b e^{-N}$ with positive constants $a, b>0$. Some results on (1.1) can be found in $[3,12,15,29,31]$.

Since the biological species compete and cooperate with each other in real world, the growth models given by patch structure systems of delay differential equation have been provided by several authors to analyze the dynamics of multiple species, see $[7,25]$ and the reference therein. Up to present, several authors in $[3,5,28]$ have researched the exponential extinction, permanence and existence of positive periodic and almost periodic solutions for the following delayed Nicholson's blowflies system with nonlinear density-dependent mortality terms and patch structure:

$$
\begin{aligned}
N_{i}^{\prime}(t)=- & D_{i i}\left(t, N_{i}(t)\right)+\sum_{j=1, j \neq i}^{n} D_{i j}\left(t, N_{j}(t)\right) \\
& +\sum_{j=1}^{l} c_{i j}(t) N_{i}\left(t-\tau_{i j}(t)\right) e^{-\gamma_{i j}(t) N_{i}\left(t-\tau_{i j}(t)\right)},
\end{aligned}
$$

where $D_{i j}(t, N)=\frac{a_{i j}(t) N}{b_{i j}(t)+N}$ or $D_{i j}(t, N)=a_{i j}(t)-b_{i j}(t) e^{-N}$.

As far as we know, few works have been done on the global exponential stability of positive almost periodic solutions for (1.2). Moreover, it is more significant to discuss the almost periodic properties of differential equations than periodic properties. Motivated by the above arguments, in this paper, we investigate the existence and global exponential stability of positive almost periodic solutions for the following delayed Nicholson's blowflies system with nonlinear density-dependent mortality terms and patch structure:

$$
\begin{aligned}
x_{i}^{\prime}(t)= & -\frac{a_{i i}(t) x_{i}(t)}{b_{i i}(t)+x_{i}(t)}+\sum_{j=1, j \neq i}^{n} \frac{a_{i j}(t) x_{j}(t)}{b_{i j}(t)+x_{j}(t)} \\
& +\sum_{j=1}^{l} \beta_{i j}(t) x_{i}\left(t-\tau_{i j}(t)\right) e^{-\gamma_{i j}(t) x_{i}\left(t-\tau_{i j}(t)\right)},
\end{aligned}
$$

where $a_{i j}, b_{i j}, \beta_{i m}, \gamma_{i m}: R \rightarrow(0,+\infty)$ and $\tau_{i m}: R \rightarrow R_{+}$are continuous almost periodic functions with $i, j=1,2, \ldots, n, m=1,2, \ldots, l$. The case of 
(1.2) with $D_{i j}(t, N)=a_{i j}(t)-b_{i j}(t) e^{-N}$ has been studied by Chen and Wang in [5] before. And it is easy to see that (1.3) is the another case of (1.2) with $D_{i j}(t, N)=\frac{a_{i j}(t) N}{b_{i j}(t)+N}$.

For convenience, we introduce some notations. Throughout this paper, given a bounded continuous function $g$ defined on $R$, let $g^{+}$and $g^{-}$be defined as

$$
g^{+}=\sup _{t \in R} g(t), \quad g^{-}=\inf _{t \in R} g(t) .
$$

It will be assumed that

$$
r_{i}=\max _{1 \leq j \leq l} \tau_{i j}^{+}>0, \quad \gamma_{i j}^{-} \geq 1, \quad i=1,2, \ldots, n, j=1,2, \ldots, l .
$$

Let $R^{n}\left(R_{+}^{n}\right)$ be the set of all (nonnegative) real vectors, we will use $x=$ $\left(x_{1}, \ldots, x_{n}\right)^{T} \in R^{n}$ to denote a column vector, in which the symbol $\left({ }^{T}\right)$ denotes the transpose of a vector. We let $|x|$ denote the absolute-value vector given by $|x|=\left(\left|x_{1}\right|, \ldots,\left|x_{n}\right|\right)^{T}$ and define $\|x\|=\max _{1 \leq i \leq n}\left|x_{i}\right|$. Denote $C=\prod_{i=1}^{n} C\left(\left[-r_{i}, 0\right], R\right)$ and $C_{+}=\prod_{i=1}^{n} C\left(\left[-r_{i}, 0\right], R_{+}\right)$as a Banach space equipped with the supremum norm defined by $\|\varphi\|=\sup _{-r_{i} \leq t \leq 0} \max _{1 \leq i \leq n}\left|\varphi_{i}(t)\right|$ for all $\varphi(t)=\left(\varphi_{1}(t), \ldots, \varphi_{n}(t)\right)^{T} \in C\left(\right.$ or $\left.\in C_{+}\right)$. If $x_{i}(t)$ is defined on $\left[t_{0}-r_{i}, v\right)$ with $t_{0}, v \in R$ and $i=1, \ldots, n$, then we define $x_{t} \in C$ as $x_{t}=\left(x_{t}^{1}, \ldots, x_{t}^{n}\right)^{T}$, where $x_{t}^{i}(\theta)=x_{t}(t+\theta)$ for all $\theta \in\left[-r_{i}, 0\right]$ and $i=1, \ldots, n$.

It is biologically reasonable to assume that only positive solutions of model (1.3) are meaningful and therefore admissible. So we consider the admissible initial conditions

$$
x_{t_{0}}=\varphi, \quad \varphi=\left(\varphi_{1}, \ldots, \varphi_{n}\right)^{T} \in C_{+} \text {and } \varphi_{i}(0)>0, i=1,2, \ldots n .
$$

We denote $x_{t}\left(t_{0}, \varphi\right)\left(x\left(t ; t_{0}, \varphi\right)\right)$ for a solution of the initial value problem (1.3) and (1.5). Also, let $\left[t_{0}, \eta(\varphi)\right)$ be the maximal right-interval of existence of $x_{t}\left(t_{0}, \varphi\right)$.

As it is easy to analyze the property of functions $\frac{1-x}{e^{x}}$ and $x e^{-x}$ in the range $R_{+}$, one can get that there exist only $\kappa \in(0,1)$ and $\tilde{\kappa} \in(1,+\infty)$ such that

$$
\frac{1-\kappa}{e^{\kappa}}=\frac{1}{e^{2}}, \quad \sup _{x \geq \kappa}\left|\frac{1-x}{e^{x}}\right|=\frac{1}{e^{2}}, \quad \kappa e^{-\kappa}=\tilde{\kappa} e^{-\tilde{\kappa}} .
$$

This paper is organized as follows: In Section 2 we give some preliminary lemmas, and in Section 3 we devote to prove our main results.

\section{Preliminary results}

In this section, some definitions and lemmas will be presented, which are of importance in proving our main results in Section 3.

Definition 1. (see $[8,11]$ ). Let $u(t): R^{1} \rightarrow R^{n}$ be continuous in $t, u(t)$ is said to be almost periodic on $R^{1}$, if for any $\varepsilon>0$, the set $T(u, \varepsilon)=\{\delta$ : $\|u(t+\delta)-u(t)\|<\varepsilon$ for all $\left.t \in R^{1}\right\}$ is relatively dense, i.e., for any $\varepsilon>0$, 
it is possible to find a real number $l=l(\varepsilon)>0$, such that for any interval with length $l(\varepsilon)$, there exists a number $\delta=\delta(\varepsilon)$ in this interval such that $\|u(t+\delta)-u(t)\|<\varepsilon$, for all $t \in R^{1}$.

From the theory of almost periodic functions in $[8,11]$, it follows that for any $\varepsilon>0$, it is possible to find a real number $l=l(\varepsilon)>0$, for any interval with length $l(\varepsilon)$, there exists a number $\delta=\delta(\varepsilon)$ in this interval such that

$$
\left\{\begin{array}{l}
\left|a_{i j}(t+\delta)-a_{i j}(t)\right|<\varepsilon, \quad\left|b_{i j}(t+\delta)-b_{i j}(t)\right|<\varepsilon, \\
\left|\beta_{i m}(t+\delta)-\beta_{i m}(t)\right|<\varepsilon, \\
\left|\tau_{i m}(t+\delta)-\tau_{i m}(t)\right|<\varepsilon, \quad\left|\gamma_{i m}(t+\delta)-\gamma_{i m}(t)\right|<\varepsilon,
\end{array}\right.
$$

for all $t \in R, i, j=1,2, \ldots, n$ and $m=1,2, \ldots, l$.

Lemma 1. Suppose that there exists a positive constant $M>\kappa$ such that

$$
\gamma_{i j}(t) \cdot M \leq \tilde{\kappa} \text { for all } t \in R, \quad i, j=1,2, \ldots, n,
$$

and for all $i=1,2, \ldots, n$,

$$
\begin{gathered}
\sup _{t \in R}\left\{-\frac{a_{i i}(t) \cdot M}{b_{i i}(t)+M}+\sum_{j=1, j \neq i}^{n} a_{i j}(t)+\frac{1}{e} \sum_{j=1}^{l} \frac{\beta_{i j}(t)}{\gamma_{i j}(t)}\right\}<0, \\
\inf _{t \in R, s \in[0, \kappa]}\left\{-\frac{a_{i i}(t)}{b_{i i}(t)+s}+\sum_{j=1, j \neq i}^{n} \frac{a_{i j}(t)}{b_{i j}(t)+M}+\sum_{j=1}^{l} \frac{\beta_{i j}(t)}{\gamma_{i j}(t)} \cdot e^{-s}\right\}>0 .
\end{gathered}
$$

Then, for $\varphi \in C^{0}=\{\varphi \mid \varphi \in C, \varphi(\theta) \in(\kappa, M)$ for all $\theta \in[-r, 0]\}$, $\eta(\varphi)=\infty \quad$ and $\quad x_{t}\left(t_{0}, \varphi\right) \in C^{0}$ for $t \geq t_{0}$.

Proof. This lemma can be proven in the similar way as in Lemma 1 of [5]. But for convenience of reading, we give the proof as follows.

Let $x(t)=x\left(t ; t_{0}, \varphi\right)=\left(x_{1}(t), x_{2}(t), \ldots, x_{n}(t)\right)^{T}$ for all $t \in\left[t_{0}, \eta(\varphi)\right)$. Firstly, we assert that

$$
x_{i}(t)>0 \text { for all } t \in\left[t_{0}, \eta(\varphi)\right), i=1,2, \ldots, n .
$$

With the reduction to absurdity, assume that there exist $s_{1} \in\left[t_{0}, \eta(\varphi)\right)$ and $i \in\{1,2, \ldots, n\}$ such that

$$
x_{i}\left(s_{1}\right)=0, \quad x_{j}(t)>0 \text { for all } t \in\left[t_{0}, s_{1}\right), j=1,2, \ldots, n .
$$

Calculating the derivative of $x_{i}(t),(1.3)$ and (2.6) imply that

$$
\begin{aligned}
0 \geq & x_{i}^{\prime}\left(s_{1}\right)=-\frac{a_{i i}\left(s_{1}\right) x_{i}\left(s_{1}\right)}{b_{i i}\left(s_{1}\right)+x_{i}\left(s_{1}\right)}+\sum_{j=1, j \neq i}^{n} \frac{a_{i j}\left(s_{1}\right) x_{j}\left(s_{1}\right)}{b_{i j}\left(s_{1}\right)+x_{j}\left(s_{1}\right)} \\
& +\sum_{j=1}^{l} \beta_{i j}\left(s_{1}\right) x_{i}\left(s_{1}-\tau_{i j}\left(s_{1}\right)\right) e^{-\gamma_{i j}\left(s_{1}\right) x_{i}\left(s_{1}-\tau_{i j}\left(s_{1}\right)\right)}=\sum_{j=1, j \neq i}^{n} \frac{a_{i j}\left(s_{1}\right) x_{j}\left(s_{1}\right)}{b_{i j}\left(s_{1}\right)+x_{j}\left(s_{1}\right)} \\
& +\sum_{j=1}^{l} \beta_{i j}\left(s_{1}\right) x_{i}\left(s_{1}-\tau_{i j}\left(s_{1}\right)\right) e^{-\gamma_{i j}\left(s_{1}\right) x_{i}\left(s_{1}-\tau_{i j}\left(s_{1}\right)\right)}>0
\end{aligned}
$$


which is paradoxical and implies that (2.5) holds.

Next we show that

$$
x_{i}(t)<M \text { for all } t \in\left[t_{0}, \eta(\varphi)\right), i=1,2, \ldots, n .
$$

Suppose, for the sake of contradiction, that (2.7) dose not hold. Then, there exist $t_{1} \in\left(t_{0}, \eta(\varphi)\right)$ and $i \in\{1,2, \ldots, n\}$ such that

$$
x_{i}\left(t_{1}\right)=M, \quad x_{j}(t)<M \text { for all } t \in\left[t_{0}-r_{i}, t_{1}\right), j=1,2, \ldots, n .
$$

Calculating the derivative of $x_{i}(t)$, together with the fact that $\sup u e^{-u}=\frac{1}{e}$, (1.3), (2.3) and (2.8) implies that

$$
\begin{aligned}
0 \leq & x_{i}^{\prime}\left(t_{1}\right)=-\frac{a_{i i}\left(t_{1}\right) x_{i}\left(t_{1}\right)}{b_{i i}\left(t_{1}\right)+x_{i}\left(t_{1}\right)}+\sum_{j=1, j \neq i}^{n} \frac{a_{i j}\left(t_{1}\right) x_{j}\left(t_{1}\right)}{b_{i j}\left(t_{1}\right)+x_{j}\left(t_{1}\right)} \\
& +\sum_{j=1}^{l} \beta_{i j}\left(t_{1}\right) x_{i}\left(t_{1}-\tau_{i j}\left(t_{1}\right)\right) e^{-\gamma_{i j}\left(t_{1}\right) x_{i}\left(t_{1}-\tau_{i j}\left(t_{1}\right)\right)} \\
= & -\frac{a_{i i}\left(t_{1}\right) x_{i}\left(t_{1}\right)}{b_{i i}\left(t_{1}\right)+x_{i}\left(t_{1}\right)}+\sum_{j=1, j \neq i}^{n} a_{i j}\left(t_{1}\right)-\sum_{j=1, j \neq i}^{n} \frac{a_{i j}\left(t_{1}\right) b_{i j}\left(t_{1}\right)}{b_{i j}\left(t_{1}\right)+x_{j}\left(t_{1}\right)} \\
& +\sum_{j=1}^{l} \beta_{i j}\left(t_{1}\right) x_{i}\left(t_{1}-\tau_{i j}\left(t_{1}\right)\right) e^{-\gamma_{i j}\left(t_{1}\right) x_{i}\left(t_{1}-\tau_{i j}\left(t_{1}\right)\right)} \\
\leq & -\frac{a_{i i}\left(t_{1}\right) M}{b_{i i}\left(t_{1}\right)+M}+\sum_{j=1, j \neq i}^{n} a_{i j}\left(t_{1}\right)+\frac{1}{e} \sum_{j=1}^{l} \frac{\beta_{i j}\left(t_{1}\right)}{\gamma_{i j}\left(t_{1}\right)} \\
\leq & \sup _{t \in R}\left\{-\frac{a_{i i}(t) M}{b_{i i}(t)+M}+\sum_{j=1, j \neq i}^{n} a_{i j}(t)+\frac{1}{e} \sum_{j=1}^{l} \frac{\beta_{i j}(t)}{\gamma_{i j}(t)}\right\}<0,
\end{aligned}
$$

which is a contradiction and implies that (2.7) holds. Then we prove that

$$
x_{i}(t)>\kappa \text { for all } t \in\left[t_{0}, \eta(\varphi)\right), i=1,2, \ldots, n .
$$

Assume, by way of contradiction, that (2.9) dose not hold. Then, there exist $t_{2} \in\left(t_{0}, \eta(\varphi)\right)$ and $i \in\{1,2, \ldots, n\}$ such that

$$
x_{i}\left(t_{2}\right)=\kappa, \quad x_{j}(t)>\kappa \text { for all } t \in\left[t_{0}-r_{i}, t_{2}\right), j=1,2, \ldots, n .
$$

From (1.4), (2.2), (2.7) and (2.10), we get

$$
\kappa \leq \gamma_{i j}\left(t_{2}\right) x_{i}\left(t_{2}-\tau_{i j}\left(t_{2}\right)\right) \leq \gamma_{i j}\left(t_{2}\right) M \leq \tilde{\kappa}
$$

and hence

$$
\gamma_{i j}\left(t_{2}\right) x_{i}\left(t_{2}-\tau_{i j}\left(t_{2}\right)\right) e^{\gamma_{i j}\left(t_{2}\right) x_{i}\left(t_{2}-\tau_{i j}\left(t_{2}\right)\right)} \geq \min \left\{\kappa e^{-\kappa}, \tilde{\kappa} e^{-\tilde{\kappa}}\right\}=\kappa e^{-\kappa},
$$

where $i, j=1,2, \ldots, n$. 
It follows from (2.4) and (2.10) that

$$
\begin{aligned}
0 \geq & x_{i}^{\prime}\left(t_{2}\right)=-\frac{a_{i i}\left(t_{2}\right) x_{i}\left(t_{2}\right)}{b_{i i}\left(t_{2}\right)+x_{i}\left(t_{2}\right)}+\sum_{j=1, j \neq i}^{n} \frac{a_{i j}\left(t_{2}\right) x_{j}\left(t_{2}\right)}{b_{i j}\left(t_{2}\right)+x_{j}\left(t_{2}\right)} \\
& +\sum_{j=1}^{l} \beta_{i j}\left(t_{2}\right) x_{i}\left(t_{2}-\tau_{i j}\left(t_{2}\right)\right) e^{-\gamma_{i j}\left(t_{2}\right) x_{i}\left(t_{2}-\tau_{i j}\left(t_{2}\right)\right)} \\
& \geq-\frac{a_{i i}\left(t_{2}\right) \kappa}{b_{i i}\left(t_{2}\right)+\kappa}+\sum_{j=1, j \neq i}^{n} \frac{a_{i j}\left(t_{2}\right) \kappa}{b_{i j}\left(t_{2}\right)+M}+\sum_{j=1}^{l} \frac{\beta_{i j}\left(t_{2}\right)}{\gamma_{i j}\left(t_{2}\right)} \kappa e^{-\kappa} \\
& \geq \kappa \inf _{t \in R, s \in[0, \kappa]}\left\{-\frac{a_{i i}(t)}{b_{i i}(t)+s}+\sum_{j=1, j \neq i}^{n} \frac{a_{i j}(t)}{b_{i j}(t)+M}+\sum_{j=1}^{l} \frac{\beta_{i j}(t)}{\gamma_{i j}(t)} e^{-s}\right\}>0,
\end{aligned}
$$

which is a contradiction and implies that (2.9) holds. Thus $x(t)$ is bounded on $\left[t_{0}, \eta(\varphi)\right)$. From Theorem 2.3.1 in [10], we easily obtain $\eta(\varphi)=+\infty$. This ends the proof of Lemma 1.

Lemma 2. Suppose that (2.2), (2.3) and (2.4) hold, and

$$
\sup _{t \in R}\left\{-\frac{a_{i i}(t) b_{i i}(t)}{\left(b_{i i}(t)+M\right)^{2}}+\sum_{j=1, j \neq i}^{n} \frac{a_{i j}(t) b_{i j}(t)}{\left(b_{i j}(t)+\kappa\right)^{2}}+\sum_{j=1}^{l} \frac{1}{e^{2}} \beta_{i j}(t)\right\}<0 .
$$

Moreover, assume that $x(t)=x\left(t ; t_{0}, \varphi\right)=\left(x_{1}(t), x_{2}(t), \ldots, x_{n}(t)\right)^{T}$ is a solution of equation (1.3) with initial condition $\varphi \in C^{0}$ and $\varphi_{i}^{\prime}$ is bounded continuous on $\left[-r_{i}, 0\right], i=1,2, \ldots, n$. Then, for any $\varepsilon>0$, there exists $l=l(\varepsilon)>0$ such that every interval $[\alpha, \alpha+l]$ contains at least one number $\delta$ for which there exists $N>0$ satisfying $\|x(t+\delta)-x(t)\| \leq \varepsilon$ for all $t>N$.

Proof. For all $i \in 1,2, \ldots, n$, define continuous functions $\Gamma_{i}(u)$ by setting

$$
\Gamma_{i}(u)=-\left[\frac{a_{i i}(t) b_{i i}(t)}{\left(b_{i i}(t)+M\right)^{2}}-u\right]+\sum_{j=1, j \neq i}^{n} \frac{a_{i j}(t) b_{i j}(t)}{\left(b_{i j}(t)+\kappa\right)^{2}}+\sum_{j=1}^{l} \frac{1}{e^{2}} \beta_{i j}(t) e^{u r_{i}}, u \in[0,1]
$$

Then, from (2.11), we have

$$
\Gamma_{i}(0)=-\frac{a_{i i}(t) b_{i i}(t)}{\left(b_{i i}(t)+M\right)^{2}}+\sum_{j=1, j \neq i}^{n} \frac{a_{i j}(t) b_{i j}(t)}{\left(b_{i j}(t)+\kappa\right)^{2}}+\sum_{j=1}^{l} \frac{1}{e^{2}} \cdot \beta_{i j}(t)<0,
$$

which implies that there exist two constants $\eta>0$ and $\lambda \in(0,1]$ such that

$$
\begin{aligned}
\Gamma_{i}(\lambda) & =-\left[\frac{a_{i i}(t) b_{i i}(t)}{\left(b_{i i}(t)+M\right)^{2}}-\lambda\right]+\sum_{j=1, j \neq i}^{n} \frac{a_{i j}(t) b_{i j}(t)}{\left(b_{i j}(t)+\kappa\right)^{2}}+\sum_{j=1}^{l} \frac{1}{e^{2}} \beta_{i j}(t) e^{\lambda r_{i}} \\
& <-\eta<0 \quad \text { for all } t \in R, i=1,2, \ldots, n .
\end{aligned}
$$


For $i \in 1,2, \ldots, n, t \in\left(-\infty, t_{0}-r_{i}\right]$, we add the definition of $x_{i}(t)$ with $x_{i}(t) \equiv x_{i}\left(t_{0}-r_{i}\right)$. Set

$$
\begin{aligned}
& \varepsilon_{i}(\delta, t)=-\left[\frac{a_{i i}(t+\delta) x_{i}(t+\delta)}{b_{i i}(t+\delta)+x_{i}(t+\delta)}-\frac{a_{i i}(t) x_{i}(t+\delta)}{b_{i i}(t+\delta)+x_{i}(t+\delta)}\right] \\
& -\left[\frac{a_{i i}(t) x_{i}(t+\delta)}{b_{i i}(t+\delta)+x_{i}(t+\delta)}-\frac{a_{i i}(t) x_{i}(t+\delta)}{b_{i i}(t)+x_{i}(t+\delta)}\right]+\sum_{j=1, j \neq i}^{n}\left[\frac{a_{i j}(t+\delta) x_{j}(t+\delta)}{b_{i j}(t+\delta)+x_{j}(t+\delta)}\right. \\
& \left.\quad-\frac{a_{i j}(t) x_{j}(t+\delta)}{b_{i j}(t+\delta)+x_{j}(t+\delta)}\right]+\sum_{j=1, j \neq i}^{n}\left[\frac{a_{i j}(t) x_{j}(t+\delta)}{b_{i j}(t+\delta)+x_{j}(t+\delta)}-\frac{a_{i j}(t) x_{j}(t+\delta)}{b_{i j}(t)+x_{j}(t+\delta)}\right] \\
& +\sum_{j=1}^{l}\left[\beta_{i j}(t+\delta)-\beta_{i j}(t)\right] x_{i}\left(t+\delta-\tau_{i j}(t+\delta)\right) e^{-\gamma_{i j}(t+\delta) x_{i}\left(t+\delta-\tau_{i j}(t+\delta)\right)} \\
& +\sum_{j=1}^{l} \beta_{i j}(t)\left[x_{i}\left(t+\delta-\tau_{i j}(t+\delta)\right) e^{-\gamma_{i j}(t+\delta) x_{i}\left(t+\delta-\tau_{i j}(t+\delta)\right)}\right. \\
& \left.\quad-x_{i}\left(t+\delta-\tau_{i j}(t)\right) e^{-\gamma_{i j}(t+\delta) x_{i}\left(t+\delta-\tau_{i j}(t)\right)}\right]+\sum_{j=1}^{l} \beta_{i j}(t)\left[x_{i}\left(t+\delta-\tau_{i j}(t)\right)\right. \\
& \left.\times e^{-\gamma_{i j}(t+\delta) x_{i}\left(t+\delta-\tau_{i j}(t)\right)}-x_{i}\left(t+\delta-\tau_{i j}(t)\right) e^{-\gamma_{i j}(t) x_{i}\left(t+\delta-\tau_{i j}(t)\right)}\right], t \in R . \quad(2.13)
\end{aligned}
$$

By Lemma 1, the solution $x(t)$ is bounded and

$$
\kappa<x_{i}(t)<M \text { for all } t \geq t_{0}, i=1,2, \ldots, n,
$$

which implies that the right-hand side of (1.3) is also bounded, and $x_{i}^{\prime}(t)$ is a bounded function on $\left[t_{0}-r_{i},+\infty\right), i=1,2, \ldots, n$. Thus, in view of the fact that $x_{i}(t) \equiv x_{i}\left(t_{0}-r_{i}\right)$ for $t \in\left(-\infty, t_{0}-r_{i}\right], i=1,2, \ldots, n$, we obtain that $x_{i}(t)$ is uniformly continuous on $R$. From (2.1) and (2.13), for any $\varepsilon$, there exists $l=l(\varepsilon)>0$, such that every interval $[\alpha, \alpha+l], \alpha \in R$, contains $\delta$ for which

$$
\left|\varepsilon_{i}(\delta, t)\right| \leq \frac{1}{2} \eta \varepsilon \quad \text { for all } t \in R, i=1,2, \ldots, n .
$$

Let $N_{0} \geq \max \left\{t_{0}, t_{0}-\delta, t_{0}+\max _{i=1,2, \ldots, n} r_{i}\right\}$ and denote $u(t)=\left(u_{1}(t), u_{2}(t), \ldots\right.$, $\left.u_{n}(t)\right)^{T}$, where $u_{i}(t)=x_{i}(t+\delta)-x_{i}(t), i=1,2, \ldots, n$. Then, for all $t \geq N_{0}$, we have

$$
\begin{aligned}
& u_{i}^{\prime}(t)=-\left[\frac{a_{i i}(t) x_{i}(t+\delta)}{b_{i i}(t)+x_{i}(t+\delta)}-\frac{a_{i i}(t) x_{i}(t)}{b_{i i}(t)+x_{i}(t)}\right]+\sum_{j=1, j \neq i}^{n}\left[\frac{a_{i i}(t) x_{j}(t+\delta)}{b_{i i}(t)+x_{j}(t+\delta)}\right. \\
& \left.-\frac{a_{i i}(t) x_{j}(t)}{b_{i i}(t)+x_{j}(t)}\right]+\sum_{j=1}^{l} \beta_{i j}(t)\left[x_{i}\left(t+\delta-\tau_{i j}(t)\right) e^{\gamma_{i j}(t) x_{i}\left(t+\delta-\tau_{i j}(t)\right)}\right. \\
& \left.-x_{i}\left(t-\tau_{i j}(t)\right) e^{-\gamma_{i j}(t) x_{i}\left(t-\tau_{i j}(t)\right)}\right]+\varepsilon_{i}(\delta, t) .
\end{aligned}
$$

Set

$$
U(t)=\left(U_{1}(t), U_{2}(t), \ldots, U_{n}(t)\right)^{T}, \text { where } U_{i}(t)=e^{\lambda t} u_{i}(t), i=1,2, \ldots, n .
$$


Let $i_{t}$ be such an index that

$$
\left|U_{i_{t}}(t)\right|=\|U(t)\|
$$

Calculating the upper left derivative of $\left|U_{i_{s}}(t)\right|$ along with (2.16), together with (1.6), (2.13), (2.14), (2.17) and the inequalities

$$
\begin{gathered}
-\left(\frac{a s}{b+s}-\frac{a t}{b+t}\right) \operatorname{sgn}(s-t)=-\frac{a b}{(b+s-\theta(s-t))^{2}}|s-t| \\
\quad \leq-\frac{a b}{(b+M)^{2}}|A-B|, \text { where } s, t \in[\kappa, M], 0<\theta<1, \\
\left|\frac{a s}{b+s}-\frac{a t}{b+t}\right|=\frac{a b}{(b+s-\theta(s-t))^{2}}|s-t| \\
\quad \leq \frac{a b}{(b+\kappa)^{2}}|s-t|, \text { where } s, t \in[\kappa,+\infty], 0<\theta<1, \\
\left|s e^{-s}-t e^{-t}\right|=\left|\frac{1-(s+\theta(t-s))}{e^{s+\theta(t-s)}}\right||s-t| \\
\leq-\frac{1}{e^{2}}|s-t|, \text { where } s, t \in[\kappa,+\infty], 0<\theta<1,
\end{gathered}
$$

we get

$$
\begin{aligned}
& \left.D^{-}\left(\left|U_{i_{s}}(s)\right|\right)\right|_{s=t} \\
& \leq \lambda e^{\lambda t}\left|u_{i_{t}}(t)\right|+e^{\lambda t}\left\{-\left[\frac{a_{i_{t} i_{t}}(t) x_{i_{t}}(t+\delta)}{b_{i_{t} i_{t}}(t)+x_{i_{t}}(t+\delta)}-\frac{a_{i_{t} i_{t}}(t) x_{i_{t}}(t)}{b_{i_{t} i_{t}}(t)+x_{i_{t}}(t)}\right]\right. \\
& \times \operatorname{sgn}\left(x_{i_{t}}(t+\delta)-x_{i_{t}}(t)\right)+\sum_{j=1, j \neq i_{t}}^{n}\left|\frac{a_{i_{t} j}(t) x_{j}(t+\delta)}{b_{i_{t} j}(t)+x_{j}(t+\delta)}-\frac{a_{i_{t} j}(t) x_{j}(t)}{b_{i_{t} j}(t)+x_{j}(t)}\right| \\
& +\sum_{j=1}^{l} \frac{\beta_{i_{t} j}(t)}{\gamma_{i_{t} j}(t)} \mid \gamma_{i_{t} j}(t) x_{i_{t}}\left(t+\delta-\tau_{i_{t} j}(t)\right) e^{-\gamma_{i_{t} j}(t) x_{i_{t}}\left(t+\delta-\tau_{i_{t} j}(t)\right)} \\
& \left.-\gamma_{i_{t} j}(t) x_{i_{t}}\left(t-\tau_{i_{t} j}(t)\right) e^{-\gamma_{i_{t} j}(t) x_{i_{t}}\left(t-\tau_{i_{t} j}(t)\right)}|+| \varepsilon_{i_{t}}(\delta, t) \mid\right\} \\
& \leq \lambda e^{\lambda t}\left|u_{i_{t}}(t)\right|+e^{\lambda t}\left\{-\frac{a_{i_{t} i_{t}}(t) b_{i_{t} i_{t}}(t)}{\left(b_{i_{t} i_{t}}(t)+M\right)^{2}}\left|u_{i_{t}}(t)\right|\right. \\
& \left.+\sum_{j=1, j \neq i_{t}}^{n} \frac{a_{i_{t} j}(t) b_{i_{t} j}(t)}{\left(b_{i_{t} j}(t)+\kappa\right)^{2}}\left|u_{j}(t)\right|+\sum_{j=1}^{l} \frac{\beta_{i_{t} j}(t)}{e^{2}}\left|u_{i_{t}}\left(t-\tau_{i_{t} j}(t)\right)\right|+\left|\varepsilon_{i_{t}}(\delta, t)\right|\right\} \\
& =-\left[\frac{a_{i_{t} i_{t}}(t) b_{i_{t} i_{t}}(t)}{\left(b_{i_{t} i_{t}}(t)+M\right)^{2}}-\lambda\right] e^{\lambda t}\left|u_{i_{t}}(t)\right|+\sum_{j=1, j \neq i_{t}}^{n} \frac{a_{i_{t} j}(t) b_{i_{t} j}(t)}{\left(b_{i_{t} j}(t)+\kappa\right)^{2}} e^{\lambda t}\left|u_{j}(t)\right| \\
& \left.+\sum_{j=1}^{l} \beta_{i_{t} j}(t) \frac{1}{e^{2}} e^{\lambda \tau_{i_{t} j}(t)} e^{\lambda\left(t-\tau_{i_{t} j}(t)\right.}\right)\left|u_{i_{t}}\left(t-\tau_{i_{t} j}(t)\right)\right|+e^{\lambda t}\left|\varepsilon_{i_{t}}(\delta, t)\right|
\end{aligned}
$$

for all $t \geq N_{0}$, which is held under the following fact:

$$
\kappa \leq \gamma_{i_{t} j}(t) x_{i_{t}}\left(t+\delta-\tau_{i_{t} j}(t)\right), \quad \gamma_{i_{t} j}(t) x_{i_{t}}^{*}\left(t-\tau_{i_{t} j}(t)\right) \leq \gamma_{i j}^{+} M \leq \tilde{\kappa},
$$


for $j=1,2, \ldots, l, t \geq N_{0}$. Let $M(t)=\max _{s \leq t}\{\|U(s)\|$. It is obvious that $M(t) \geq$ $\|U(t)\|$ and $M(t)$ is non-decreasing. Now, we distinguish two cases to finish the proof.

\section{Case1.}

$$
M(t)>\|U(t)\| \text { for all } t \geq N_{0}
$$

We claim that

$$
M(t) \equiv M\left(N_{0}\right) \quad \text { is a constant for all } t \geq N_{0} .
$$

Assume, by a way of contradiction, that (2.24) does not hold. Then there exists $\tilde{t_{1}}>N_{0}$ such that $M\left(\tilde{t_{1}}\right)>M\left(N_{0}\right)$. Since

$$
M\left(N_{0}\right) \geq\|U(t)\| \quad \text { for all } t \geq N_{0} .
$$

There must exist $\beta \in\left(N_{0}, \tilde{t_{1}}\right)$ such that

$$
\|U(\beta)\|=M\left(\tilde{t_{1}}\right) \geq M(\beta),
$$

which contradicts (2.23) and implies that (2.24) holds. It follows that there exists $\tilde{t_{2}}>N_{0}$ such that

$$
\|u(t)\| \leq e^{-\lambda t} M(t)=e^{-\lambda t} M\left(N_{0}\right)<\varepsilon \quad \text { for all } t \geq \tilde{t_{2}} .
$$

Case 2. There is $\rho>N_{0}$ such that $M(\rho)=\|U(\rho)\|$. Then, in view of (2.12), (2.15) and (2.22), we have

$$
\begin{aligned}
0 \leq & \left.D^{-}\left(\left|U_{i_{s}}(s)\right|\right)\right|_{s=\rho} \leq-\left[\frac{a_{i_{\rho} i_{\rho}}(\rho) b_{i_{\rho} i_{\rho}}(\rho)}{\left(b_{i_{\rho} i_{\rho}}(\rho)+M\right)^{2}}-\lambda\right] e^{\lambda \rho}\left|U_{i_{\rho}}(\rho)\right| \\
& +\sum_{j=1, j \neq i_{\rho}}^{n} \frac{a_{i_{\rho} j}(\rho) b_{i_{\rho} j}(\rho)}{\left(b_{i_{\rho} j}(\rho)+\kappa\right)^{2}} e^{\lambda \rho}\left|U_{j}(\rho)\right|+\sum_{j=1}^{l} \beta_{i_{\rho} j}(\rho) \frac{1}{e^{2}} e^{\lambda \tau_{i_{\rho} j}(\rho)} \\
& \left.\times e^{\lambda\left(\rho-\tau_{i_{\rho} j}(\rho)\right.}\right)\left|U_{i_{\rho}}\left(\rho-\tau_{i_{\rho} j}(\rho)\right)\right|+e^{\lambda \rho}\left|\varepsilon_{i_{\rho}}(\delta, \rho)\right| \\
& \leq\left\{-\left[\frac{a_{i_{\rho} i_{\rho}}(\rho) b_{i_{\rho} i_{\rho}}(\rho)}{\left(b_{i_{\rho} i_{\rho}}(\rho)+M\right)^{2}}-\lambda\right]+\sum_{j=1, j \neq i_{\rho}}^{n} \frac{a_{i_{\rho} j}(\rho) b_{i_{\rho} j}(\rho)}{\left(b_{i_{\rho} j}(\rho)+\kappa\right)^{2}}\right. \\
& \left.+\sum_{j=1}^{l} \frac{1}{e^{2}} \beta_{i_{\rho} j}(\rho) e^{\lambda r_{i_{\rho}}}\right\}\|U(\rho)\|+\frac{1}{2} \eta \varepsilon e^{\lambda \rho}<-\eta\|U(\rho)\|+\eta \varepsilon e^{\lambda \rho},
\end{aligned}
$$

which yields that

$$
e^{\lambda \rho}\|u(\rho)\|=\|U(\rho)\|<\varepsilon e^{\lambda \rho} \quad \text { and } \quad\|u(\rho)\|<\varepsilon .
$$

For any $t>\rho$, with the same approach as that in deriving of (2.25), we show

$$
e^{\lambda t}\|u(t)\|=\|U(t)\|<\varepsilon e^{\lambda t} \quad \text { and } \quad\|u(t)\|<\varepsilon,
$$

if $M(t)=\|U(t)\|$. On the other hand, if $M(t)>\|U(t)\|$ and $t>\rho$, we can choose $\rho \leq t_{3}<t$ such that

$$
M\left(t_{3}\right)=\left\|U\left(t_{3}\right)\right\|, \quad \text { and } \quad M(s)>\|U(s)\| \quad \text { for all } t \in\left(t_{3}, t\right],
$$


which together with $(2.26)$ yields $\left\|u\left(t_{3}\right)\right\|<\varepsilon$.

With a similar argument as that in the proof of case one, we can show that

$$
M(s) \equiv M\left(t_{3}\right) \quad \text { is a constant for all } s \in\left(t_{3}, t\right],
$$

which implies that

$$
\|u(t)\|<e^{-\lambda t} M(t)=e^{-\lambda t} M\left(t_{3}\right)=\left\|u\left(t_{3}\right)\right\| e^{-\lambda\left(t-t_{3}\right)}<\varepsilon .
$$

In summary, there must exist $N>\max \left\{\rho, N_{0}, \tilde{t_{2}}\right\}$ such that $\|u(t)\| \leq \varepsilon$ holds for all $t>N$. This completes the proof.

\section{Main results}

In this section, we establish sufficient conditions for the existence and global exponential stability of positive almost periodic solutions for system (1.3).

Theorem 1. Under the assumptions of Lemma 2, system (1.3) has at least one positive almost periodic solution $x^{*}(t)$.

Proof. Let $v(t)=v\left(t ; t_{0}, \varphi\right)=\left(v_{1}(t), v_{2}(t), \ldots, v_{n}(t)\right)^{T}$ be a solution of system (1.3) with initial conditions satisfying the assumptions in Lemma 2. We also add the definition of $v(t)$ with $v_{i}(t) \equiv v_{i}\left(t_{0}-r_{i}\right)$ for all $t \in\left(-\infty, t_{0}-r_{i}\right]$, $i=1,2, \ldots, n$.

For all $t \in R, i=1,2, \ldots, n$, set

$$
\begin{aligned}
\varepsilon_{i, k}(t) & =-\left[\frac{a_{i i}\left(t+t_{k}\right) x_{i}\left(t+t_{k}\right)}{b_{i i}\left(t+t_{k}\right)+x_{i}\left(t+t_{k}\right)}-\frac{a_{i i}(t) x_{i}\left(t+t_{k}\right)}{b_{i i}\left(t+t_{k}\right)+x_{i}\left(t+t_{k}\right)}\right] \\
& -\left[\frac{a_{i i}(t) x_{i}\left(t+t_{k}\right)}{b_{i i}\left(t+t_{k}\right)+x_{i}\left(t+t_{k}\right)}-\frac{a_{i i}(t) x_{i}\left(t+t_{k}\right)}{b_{i i}(t)+x_{i}\left(t+t_{k}\right)}\right] \\
& +\sum_{j=1, j \neq i}^{n}\left[\frac{a_{i j}\left(t+t_{k}\right) x_{j}\left(t+t_{k}\right)}{b_{i j}\left(t+t_{k}\right)+x_{j}\left(t+t_{k}\right)}-\frac{a_{i j}(t) x_{j}\left(t+t_{k}\right)}{b_{i j}\left(t+t_{k}\right)+x_{j}\left(t+t_{k}\right)}\right] \\
& +\sum_{j=1, j \neq i}^{n}\left[\frac{a_{i j}(t) x_{j}\left(t+t_{k}\right)}{b_{i j}\left(t+t_{k}\right)+x_{j}\left(t+t_{k}\right)}-\frac{a_{i j}(t) x_{j}\left(t+t_{k}\right)}{b_{i j}(t)+x_{j}\left(t+t_{k}\right)}\right] \\
+ & \sum_{j=1}^{l}\left[\beta_{i j}\left(t+t_{k}\right)-\beta_{i j}(t)\right] x_{i}\left(t+t_{k}-\tau_{i j}\left(t+t_{k}\right)\right) e^{-\gamma_{i j}\left(t+t_{k}\right) x_{i}\left(t+t_{k}-\tau_{i j}\left(t+t_{k}\right)\right)} \\
& +\sum_{j=1}^{l} \beta_{i j}(t)\left[x_{i}\left(t+t_{k}-\tau_{i j}\left(t+t_{k}\right)\right) e^{-\gamma_{i j}\left(t+t_{k}\right) x_{i}\left(t+t_{k}-\tau_{i j}\left(t+t_{k}\right)\right)}\right. \\
& \left.-x_{i}\left(t+t_{k}-\tau_{i j}(t)\right) e^{-\gamma_{i j}\left(t+t_{k}\right) x_{i}\left(t+t_{k}-\tau_{i j}(t)\right)}\right] \\
& +\sum_{j=1}^{l} \beta_{i j}(t)\left[x_{i}\left(t+t_{k}-\tau_{i j}(t)\right) e^{-\gamma_{i j}(t+\delta) x_{i}\left(t+\delta-\tau_{i j}(t)\right)}\right. \\
& \left.-x_{i}\left(t+\delta-\tau_{i j}(t)\right) e^{-\gamma_{i j}(t) x_{i}\left(t+t_{k}-\tau_{i j}(t)\right)}\right]
\end{aligned}
$$


where $\left\{t_{k}\right\}$ is any sequence of real numbers. By Lemma 1, the solution $v(t)$ is bounded and

$$
\kappa<v_{i}(t)<M \text { for all } t \in R, \quad i=1,2, \ldots, n,
$$

which implies that the right-hand side of (1.3) is also bounded, and $v_{i}^{\prime}(t)(i=$ $1,2, \ldots, n)$ are bounded functions on $\left[t_{0}-r_{i},+\infty\right)$. Thus, in view of the fact that $v_{i}(t) \equiv v_{i}\left(t_{0}-r_{i}\right)$ for all $t \in\left(-\infty, t_{0}-r_{i}\right], i=1,2, \ldots, n$, we obtain that $v_{i}(t)(i=1,2, \ldots, n)$ are uniformly continuous on $R$. Then, from the almost periodicity of $a_{i j}, b_{i j}, \beta_{i m}, \gamma_{i m}$ and $\tau_{i m}$, we can select a sequence $\left\{t_{k}\right\} \rightarrow+\infty$ such that

$$
\begin{cases}\left|a_{i j}\left(t+t_{k}\right)-a_{i j}(t)\right| \leq \frac{1}{k}, & \left|b_{i j}\left(t+t_{k}\right)-b_{i j}(t)\right| \leq \frac{1}{k} \\ \left|\beta_{i m}\left(t+t_{k}\right)-\beta_{i m}(t)\right| \leq \frac{1}{k}, & \\ \left|\tau_{i m}\left(t+t_{k}\right)-\tau_{i m}(t)\right| \leq \frac{1}{k}, & \left|\gamma_{i m}\left(t+t_{k}\right)-\gamma_{i m}(t)\right| \leq \frac{1}{k}\end{cases}
$$

for all $t \in R, i, j=1,2, \ldots, n$ and $m=1,2, \ldots, l$.

Since $\left\{v_{i}\left(t+t_{k}\right)\right\}_{k=1}^{+\infty}(i=1,2, \ldots, n)$ and $\left\{v_{i}^{\prime}\left(t+t_{k}\right)\right\}_{k=1}^{+\infty}(i=1,2, \ldots, n)$ are uniformly bounded, then $\left\{v_{i}\left(t+t_{k}\right)\right\}_{k=1}^{+\infty}(i=1,2, \ldots, n)$ is uniformly bounded and equi-uniformly continuous, by the Arzel-Ascoli lemma and the diagonal selection principle, we can choose a subsequence $\left\{t_{k_{j}}\right\}$ of $\left\{t_{k}\right\}$ such that $v_{i}(t+$ $t_{k_{j}}$ ) (for convenience, we still denote it by $v_{i}\left(t+t_{k}\right)(i=1,2, \ldots, n)$ ) uniformly converges to a continuous function $x_{i}^{*}(t)(i=1,2, \ldots, n)$ on any compact set of $R$, and

$$
\kappa \leq x_{i}^{*}(t) \leq M \text { for all } t \in R, i=1,2, \ldots, n .
$$

Now, we prove that $x^{*}(t)=\left(x_{1}^{*}(t), x_{2}^{*}(t), \ldots, x_{n}^{*}(t)\right)^{T}$ is a solution of (1.3). In fact, for any $t \geq t_{0}$ and $\Delta t \in R$, from (3.1), we have

$$
\begin{aligned}
& x_{i}^{*}(t+\Delta t)-x_{i}^{*}(t)=\lim _{k \rightarrow+\infty}\left[v_{i}\left(t+\Delta t+t_{k}\right)-v_{i}\left(t+t_{k}\right)\right] \\
& =\lim _{k \rightarrow+\infty} \int_{t}^{t+\Delta t}\left\{-\frac{a_{i i}\left(\mu+t_{k}\right) v_{i}\left(\mu+t_{k}\right)}{b_{i i}\left(\mu+t_{k}\right)+v_{i}\left(\mu+t_{k}\right)}+\sum_{j=1, j \neq i}^{n} \frac{a_{i j}\left(\mu+t_{k}\right) v_{j}\left(\mu+t_{k}\right)}{b_{i j}\left(\mu+t_{k}\right)+v_{j}\left(\mu+t_{k}\right)}\right. \\
& \left.\quad+\sum_{j=1}^{l} \beta_{i j}\left(\mu+t_{k}\right) v_{i}\left(\mu+t_{k}-\tau_{i j}\left(\mu+t_{k}\right)\right) e^{-\gamma_{i j}\left(\mu+t_{k}\right) v_{i}\left(\mu+t_{k}-\tau_{i j}\left(\mu+t_{k}\right)\right)}\right\} d \mu \\
& =\lim _{k \rightarrow+\infty} \int_{t}^{t+\Delta t}\left\{-\frac{a_{i i}(\mu) v_{i}\left(\mu+t_{k}\right)}{b_{i i}(\mu)+v_{i}\left(\mu+t_{k}\right)}+\sum_{j=1, j \neq i}^{n} \frac{a_{i j}(\mu) v_{j}\left(\mu+t_{k}\right)}{b_{i j}(\mu)+v_{j}\left(\mu+t_{k}\right)}\right. \\
& \left.\quad+\sum_{j=1}^{l} \beta_{i j}(\mu) v_{i}\left(\mu+t_{k}-\tau_{i j}(\mu)\right) e^{-\gamma_{i j}(\mu) v_{i}\left(\mu+t_{k}-\tau_{i j}(\mu)\right)}+\varepsilon_{i, k}(\mu)\right\} d \mu \\
& =\int_{t}^{t+\Delta t}\left\{-\frac{a_{i i}(\mu) x_{i}^{*}(\mu)}{b_{i i}(\mu)+x_{i}^{*}(\mu)}+\sum_{j=1, j \neq i}^{n} \frac{a_{i j}(\mu) x_{j}^{*}(\mu)}{b_{i j}(\mu)+x_{j}^{*}(\mu)}\right. \\
& \left.+\sum_{j=1}^{l} \beta_{i j}(\mu) x_{i}^{*}\left(\mu-\tau_{i j}(\mu)\right) e^{-\gamma_{i j}(\mu) x_{i}^{*}\left(\mu-\tau_{i j}(\mu)\right)}\right\} d \mu
\end{aligned}
$$


where $t+\Delta t \geq t_{0}, i=1,2, \ldots, n$. Consequently, (3.2) implies that

$$
\begin{aligned}
& \frac{d}{d t}\left\{x_{i}^{*}(t)\right\}=-\frac{a_{i i}(t) x_{i}^{*}(t)}{b_{i i}(t)+x_{i}^{*}(t)}+\sum_{j=1, j \neq i}^{n} \frac{a_{i j}(t) x_{j}^{*}(t)}{b_{i j}(t)+x_{j}^{*}(t)} \\
& \quad+\sum_{j=1}^{l} \beta_{i j}(t) x_{i}^{*}\left(t-\tau_{i j}(t)\right) e^{-\gamma_{i j}(t) x_{i}^{*}\left(t-\tau_{i j}(t)\right)}, \quad i=1,2, \ldots, n .
\end{aligned}
$$

Therefore, $x_{i}^{*}(t)$ is a solution of (1.3).

Next we prove that $x_{i}^{*}(t)$ is an almost periodic solution of (1.3). From Lemma 2, for any $\varepsilon>0$, there exists $l=l(\varepsilon)>0$, such that every interval $[\alpha, \alpha+l]$ contains at least one number $\delta$ for which there exists $N>0$ satisfying

$$
|v(t+\delta)-v(t)| \leq \varepsilon \text { for all } t>N
$$

Then, for any fixed $s \in R$, we can find a sufficiently large positive integer $N_{1}>N$ such that for any $k>N_{1}$,

$$
s+t_{k}>N, \quad\left|v\left(s+t_{k}+\delta\right)-v\left(s+t_{k}\right)\right| \leq \varepsilon .
$$

Let $k \rightarrow+\infty$, we obtain

$$
\left|x^{*}(s+\delta)-x^{*}(s)\right| \leq \varepsilon,
$$

which implies that $x^{*}(t)$ is an almost periodic solution of system (1.3). The proof of Theorem 1 is now complete.

Theorem 2. Suppose that all conditions in Theorem 1 are satisfied. Let $x^{*}(t)$ be the positive almost periodic solution of equation (1.3) in Theorem 1. Then, $x^{*}(t)$ is globally exponentially stable, i.e., the solution $x\left(t ; t_{0}, \varphi\right)$ of $(1.3)$ with admissible initial conditions (1.5) converges exponentially to $x^{*}(t)$ as $t \rightarrow+\infty$.

Proof. Let $x^{*}(t)$ be the positive almost periodic solution of equation (1.3) in Theorem 1 . To prove Theorem 2, we should show the global exponential stability for $x^{*}(t)$. Since $\varphi \in C_{+}$, using Theorem 5.2.1 in [23], we have $x_{t}\left(t_{0}, \varphi\right) \in C_{+}$ for all $t \in\left[t_{0}, \eta(\varphi)\right)$. Let $x(t)=x\left(t ; t_{0}, \varphi\right)=\left(x_{1}(t), x_{2}(t), \ldots, x_{n}(t)\right)^{T}$ for all $t \in\left[t_{0}, \eta(\varphi)\right)$.

Firstly, we show that there is $t_{\varphi}>t_{0}$ such that

$$
\kappa<x_{i}(t)<M \text { for all } t \geq t_{\varphi}, \quad i=1,2, \ldots, n \text {. }
$$

We next show that there exists $t_{4} \in\left[t_{0},+\infty\right)$ such that

$$
x_{i}\left(t_{4}\right)<M, \quad i=1,2, \ldots, n .
$$

Otherwise, there exists $i \in\{1,2, \ldots, n\}$ such that

$$
x_{i}(t) \geq M \text { for all } t \in\left[t_{0},+\infty\right),
$$


which together with the fact that $\sup _{u \geq 0} u e^{-u}=\frac{1}{e},(1.3),(2.3)$ and (3.5) implies that

$$
\begin{aligned}
x_{i}^{\prime}(t) & =-\frac{a_{i i}(t) x_{i}(t)}{b_{i i}(t)+x_{i}(t)}+\sum_{j=1, j \neq i}^{n} \frac{a_{i j}(t) x_{j}(t)}{b_{i j}(t)+x_{j}(t)} \\
& +\sum_{j=1}^{l} \beta_{i j}(t) x_{i}\left(t-\tau_{i j}(t)\right) e^{-\gamma_{i j}(t) x_{i}\left(t-\tau_{i j}(t)\right)} \\
& =-\frac{a_{i i}(t) x_{i}(t)}{b_{i i}(t)+x_{i}(t)}+\sum_{j=1, j \neq i}^{n} a_{i j}(t)-\sum_{j=1, j \neq i}^{n} \frac{a_{i j}(t) b_{i j}(t)}{b_{i j}(t)+x_{j}(t)} \\
& +\sum_{j=1}^{l} \beta_{i j}(t) x_{i}\left(t-\tau_{i j}(t)\right) e^{-\gamma_{i j}(t) x_{i}\left(t-\tau_{i j}(t)\right)} \\
& \leq-\frac{a_{i i}(t) \cdot M}{b_{i i}(t)+M}+\sum_{j=1, j \neq i}^{n} a_{i j}(t)+\frac{1}{e} \sum_{j=1}^{l} \frac{\beta_{i j}(t)}{\gamma_{i j}(t)}<0 \text { for all } t \geq t_{0} .
\end{aligned}
$$

It leads to

$$
\begin{aligned}
x_{i}(t) & =x_{i}\left(t_{0}\right)+\int_{t_{0}}^{t} x_{i}^{\prime}(s) d s \\
& \leq x_{i}\left(t_{0}\right)+\sup _{t \in R}\left\{-\frac{a_{i i}(t) \cdot M}{b_{i i}(t)+M}+\sum_{j=1, j \neq i}^{n} a_{i j}(t)+\frac{1}{e} \sum_{j=1}^{l} \frac{\beta_{i j}(t)}{\gamma_{i j}(t)}\right\} \times\left(t-t_{0}\right)
\end{aligned}
$$

for all $t \geq t_{0}$. Thus

$$
\lim _{t \rightarrow+\infty} x_{i}(t)=-\infty
$$

which contradicts with (2.5). Hence, (3.4) holds. We claim

$$
x_{i}(t)<M \text { for all } t \in\left[t_{0},+\infty\right), \quad i=1,2, \ldots, n .
$$

Suppose, for the sake of contradiction, there exists $t_{5} \in\left[t_{4},+\infty\right)$ and $i \in$ $\{1,2, \ldots, n\}$ such that

$$
x_{i}\left(t_{5}\right)=M, \quad x_{j}(t)<M \text { for all } t \in\left[t_{4}, t_{5}\right), j=1,2, \ldots, n .
$$

Calculating the derivative of $x_{i}(t)$, together with the fact that $\sup _{u \geq 0} u e^{-u}=\frac{1}{e}$, (1.3), (2.3) and (3.7) imply that

$$
\begin{aligned}
0 \leq x_{i}^{\prime}\left(t_{5}\right) & =-\frac{a_{i i}\left(t_{5}\right) x_{i}\left(t_{5}\right)}{b_{i i}\left(t_{5}\right)+x_{i}\left(t_{5}\right)}+\sum_{j=1, j \neq i}^{n} \frac{a_{i j}\left(t_{5}\right) x_{j}\left(t_{5}\right)}{b_{i j}\left(t_{5}\right)+x_{j}\left(t_{5}\right)} \\
& +\sum_{j=1}^{l} \beta_{i j}\left(t_{5}\right) x_{i}\left(t_{5}-\tau_{i j}\left(t_{5}\right)\right) e^{-\gamma_{i j}\left(t_{5}\right) x_{i}\left(t_{5}-\tau_{i j}\left(t_{5}\right)\right)} \\
= & -\frac{a_{i i}\left(t_{5}\right) x_{i}\left(t_{5}\right)}{b_{i i}\left(t_{5}\right)+x_{i}\left(t_{5}\right)}+\sum_{j=1, j \neq i}^{n} a_{i j}\left(t_{5}\right)-\sum_{j=1, j \neq i}^{n} \frac{a_{i j}\left(t_{5}\right) b_{i j}\left(t_{5}\right)}{b_{i j}\left(t_{5}\right)+x_{j}\left(t_{5}\right)}
\end{aligned}
$$




$$
\begin{array}{r}
+\sum_{j=1}^{l} \beta_{i j}\left(t_{5}\right) x_{i}\left(t_{5}-\tau_{i j}\left(t_{5}\right)\right) e^{-\gamma_{i j}\left(t_{5}\right) x_{i}\left(t_{5}-\tau_{i j}\left(t_{5}\right)\right)} \\
\leq-\frac{a_{i i}\left(t_{5}\right) M}{b_{i i}\left(t_{5}\right)+M}+\sum_{j=1, j \neq i}^{n} a_{i j}\left(t_{5}\right)+\frac{1}{e} \sum_{j=1}^{l} \frac{\beta_{i j}\left(t_{5}\right)}{\gamma_{i j}\left(t_{5}\right)}<0,
\end{array}
$$

which is a contradiction and implies that (3.6) holds.

Furthermore, we show that $l_{i}=\liminf _{t \rightarrow+\infty} x_{i}(t)>\kappa$ for all $i=1,2, \ldots, n$. By a way of contradiction, we assume that there exists $i \in\{1,2, \ldots, n\}$ such that $0 \leq l_{i} \leq \kappa$. By the fluctuation lemma [22], there exists a sequence $\left\{t_{k}\right\}_{k \geq 1}$ such that

$$
t_{k} \rightarrow+\infty, \quad x_{i}\left(t_{k}\right) \rightarrow \liminf _{t \rightarrow+\infty} x_{i}(t), \quad x_{i}^{\prime}\left(t_{k}\right) \rightarrow 0 \quad \text { as } k \rightarrow+\infty .
$$

Since $\left\{x_{t_{k}}\right\}_{k \geq 1}$ is bounded and equicontinuous, by the Ascoli-Arzel theorem, there exists a subsequence, still denoted by itself for simplicity of notation, such that

$$
x_{t_{k}} \rightarrow \varphi^{*} \text { for some } \varphi^{*} \in C_{+}
$$

Moreover,

$$
\varphi_{j}^{*}(0)=l_{j} \leq \varphi_{j}^{*}(\theta) \leq M \text { for } \theta \in\left[-r_{j}, 0\right), j=1,2, \ldots n .
$$

Without loss of generality, we assume that all $a_{i j}\left(t_{k}\right), b_{i j}\left(t_{k}\right), \beta_{i m}\left(t_{k}\right)$, $\gamma_{i m}\left(t_{k}\right)$ and $\tau_{i m}\left(t_{k}\right)$ are convergent to $a_{i j}^{*}, b_{i j}^{*}, \beta_{i m}^{*}, \gamma_{i m}^{*}$ and $\tau_{i m}^{*}$, respectively, where $i, j=1,2, \ldots, n, m=1,2, \ldots, l$. This can be achieved because of almost periodicity. Then by (1.5) and (2.2) we arrive at

$$
l_{i} \leq \gamma_{i j}^{*} \varphi_{i}^{*}\left(-\tau_{i j}^{*}\right) \leq \gamma_{i j}^{*} M \leq \tilde{\kappa}, j=1,2, \ldots, l .
$$

It follows from

$$
\begin{aligned}
x_{i}^{\prime}\left(t_{k}\right)=- & \frac{a_{i i}\left(t_{k}\right) x_{i}\left(t_{k}\right)}{b_{i i}\left(t_{k}\right)+x_{i}\left(t_{k}\right)}+\sum_{j=1, j \neq i}^{n} \frac{a_{i j}\left(t_{k}\right) x_{j}\left(t_{k}\right)}{b_{i j}\left(t_{k}\right)+x_{j}\left(t_{k}\right)} \\
& +\sum_{j=1}^{l} \beta_{i j}\left(t_{k}\right) x_{i}\left(t_{k}-\tau_{i j}\left(t_{k}\right)\right) e^{-\gamma_{i j}\left(t_{k}\right) x_{i}\left(t_{k}-\tau_{i j}\left(t_{k}\right)\right)}
\end{aligned}
$$

that (taking limits)

$$
\begin{aligned}
& 0=-\frac{a_{i i}^{*} l_{i}}{b_{i i}^{*}+l_{i}}+\sum_{j=1, j \neq i}^{n} \frac{a_{i j}^{*} \varphi_{j}^{*}(0)}{b_{i j}^{*}+\varphi_{j}^{*}(0)}+\sum_{j=1}^{l} \beta_{i j}^{*} \varphi_{i}^{*}\left(-\tau_{i j}^{*}\right) e^{-\gamma_{i j}^{*} \varphi_{i}^{*}\left(-\tau_{i j}^{*}\right)} \\
& \geq-\frac{a_{i i}^{*} \cdot l_{i}}{b_{i i}^{*}+l_{i}}+\sum_{j=1, j \neq i}^{n} \frac{a_{i j}^{*} \cdot l_{i}}{b_{i j}^{*}+M}+\sum_{j=1}^{l} \frac{\beta_{i j}^{*}}{\gamma_{i j}^{*}} \cdot l_{i} e^{-l_{i}} \\
& \geq \quad l_{i} \inf _{t \in R, s \in[0, \kappa]}\left\{-\frac{a_{i i}(t)}{b_{i i}(t)+s}+\sum_{j=1, j \neq i}^{n} \frac{a_{i j}(t)}{b_{i j}(t)+M}+\sum_{j=1}^{l} \frac{\beta_{i j}(t)}{\gamma_{i j}(t)} e^{-s}\right\}>0,
\end{aligned}
$$


which is a contradiction. This proves that $l_{i}>\kappa$ for all $i=1,2, \ldots n$.

Finally, we prove that $x^{*}(t)$ is globally exponentially stable.

Let $y(t)=x(t)-x^{*}(t)=\left(y_{1}(t), y_{2}(t), \ldots, y_{n}(t)\right)^{T}$, where $y_{i}(t)=x_{i}(t)-$ $x_{i}^{*}(t), t \in\left[t_{0}-r_{i},+\infty\right), i=1,2, \ldots, n$. Then

$$
\begin{aligned}
y_{i}^{\prime}(t) & =-\left[\frac{a_{i i}(t) x_{i}(t)}{b_{i i}(t)+x_{i}(t)}-\frac{a_{i i}(t) x_{i}^{*}(t)}{b_{i i}(t)+x_{i}^{*}(t)}\right]+\sum_{j=1, j \neq i}^{n}\left[\frac{a_{i j}(t) x_{j}(t)}{b_{i j}(t)+x_{j}(t)}\right. \\
& \left.-\frac{a_{i j}(t) x_{j}^{*}(t)}{b_{i j}(t)+x_{j}^{*}(t)}\right]+\sum_{j=1}^{l} \beta_{i j}(t)\left[x_{i}\left(t-\tau_{i j}(t)\right) e^{-\gamma_{i j}(t) x_{i}\left(t-\tau_{i j}(t)\right)}\right. \\
& \left.-x_{i}^{*}\left(t-\tau_{i j}(t)\right) e^{-\gamma_{i j}(t) x_{i}^{*}\left(t-\tau_{i j}(t)\right)}\right] .
\end{aligned}
$$

It follows from (3.3) that there exists $t_{\varphi, x^{*}}>t_{0}$ such that

$$
\kappa \leq x_{i}(t), \quad x_{i}^{*}(t) \leq M \text { for all } t \in\left[t_{\varphi, x^{*}}-r_{i},+\infty\right), i=1,2, \ldots, n .
$$

We consider the Lyapunov functional

$$
V_{i}(t)=\left|y_{i}(t)\right| e^{\lambda t}, \text { where } \lambda \text { is defined in }(2.12), i=1,2, \ldots, n \text {. }
$$

Calculating the upper left derivative of $V_{i}(t)$ along with the solution $y_{i}(t)$ of (3.8). We have

$$
\begin{aligned}
& D^{-}\left(V_{i}(t)\right) \leq \lambda\left|y_{i}(t)\right| e^{\lambda t}-\left[\frac{a_{i i}(t) x_{i}(t)}{b_{i i}(t)+x_{i}(t)}-\frac{a_{i i}(t) x_{i}^{*}(t)}{b_{i i}(t)+x_{i}^{*}(t)}\right] \\
& \quad \times \operatorname{sgn}\left(x_{i}(t)-x_{i}^{*}(t)\right) e^{\lambda t}+\sum_{j=1, j \neq i}^{n}\left|\frac{a_{i j}(t) x_{j}(t)}{b_{i j}(t)+x_{j}(t)}-\frac{a_{i j}(t) x_{j}^{*}(t)}{b_{i j}(t)+x_{j}^{*}(t)}\right| e^{\lambda t}+\sum_{j=1}^{l} \beta_{i j}(t) \\
& \quad \times\left|x_{i}\left(t-\tau_{i j}(t)\right) e^{-\gamma_{i j}(t) x_{i}\left(t-\tau_{i j}(t)\right)}-x_{i}^{*}\left(t-\tau_{i j}(t)\right) e^{-\gamma_{i j}(t) x_{i}^{*}\left(t-\tau_{i j}(t)\right)}\right| e^{\lambda t}
\end{aligned}
$$

In the sequel, we claim that

$$
\begin{aligned}
V_{i}(t) & =\left|y_{i}(t)\right| e^{\lambda t}<e^{\lambda t_{\varphi, x^{*}}}\left(\max _{i=1,2, \ldots, n} \max _{t \in\left[t_{0}-r_{i}, t_{\varphi, x^{*}}\right]}\left|x(t)-x^{*}(t)\right|+1\right) \\
& :=K_{\varphi, x^{*}} \text { for all } t>t_{\varphi, x^{*}}, i=1,2, \ldots, n
\end{aligned}
$$

Contrarily, there must exist $t_{*}>t_{\varphi, x^{*}}$ and $i \in\{1,2, \ldots, n\}$ such that

$$
V_{i}\left(t_{*}\right)=K_{\varphi, x^{*}}, V_{j}(t)<K_{\varphi, x^{*}} \quad \text { for all } t \in\left[t_{0}-r_{j}, t_{*}\right), j=1,2, \ldots, n \text {. }
$$

Since

$\kappa \leq \gamma_{i j}\left(t_{*}\right) x_{i}\left(t_{*}-\tau_{i j}\left(t_{*}\right)\right), \gamma_{i j}\left(t_{*}\right) x_{i}^{*}\left(t_{*}-\tau_{i j}\left(t_{*}\right)\right) \leq \gamma_{i j}^{+} M \leq \tilde{\kappa}, \quad j=1,2, \ldots, l$, together with (2.18)-(2.21), (3.9), (3.10) and (3.12), we get

$$
0 \leq D^{-}\left(V_{i}\left(t_{*}\right)\right) \leq \lambda\left|y_{i}\left(t_{*}\right)\right| e^{\lambda t_{*}}-\left[\frac{a_{i i}\left(t_{*}\right) x_{i}\left(t_{*}\right)}{b_{i i}\left(t_{*}\right)+x_{i}\left(t_{*}\right)}-\frac{a_{i i}\left(t_{*}\right) x_{i}^{*}\left(t_{*}\right)}{b_{i i}\left(t_{*}\right)+x_{i}^{*}\left(t_{*}\right)}\right]
$$




$$
\begin{aligned}
\times & \operatorname{sgn}\left(x_{i}\left(t_{*}\right)-x_{i}^{*}\left(t_{*}\right)\right) e^{\lambda t_{*}}+\sum_{j=1, j \neq i}^{n}\left|\frac{a_{i j}\left(t_{*}\right) x_{j}\left(t_{*}\right)}{b_{i j}\left(t_{*}\right)+x_{j}\left(t_{*}\right)}-\frac{a_{i j}\left(t_{*}\right) x_{j}^{*}\left(t_{*}\right)}{b_{i j}\left(t_{*}\right)+x_{j}^{*}\left(t_{*}\right)}\right| e^{\lambda t_{*}} \\
& +\sum_{j=1}^{l} \frac{\beta_{i j}\left(t_{*}\right)}{\gamma_{i j}\left(t_{*}\right)} \mid \beta_{i j}\left(t_{*}\right) x_{i}\left(t_{*}-\tau_{i j}\left(t_{*}\right)\right) e^{-\gamma_{i j}\left(t_{*}\right) x_{i}\left(t_{*}-\tau_{i j}\left(t_{*}\right)\right)} \\
& -\beta_{i j}\left(t_{*}\right) x_{i}^{*}\left(t_{*}-\tau_{i j}\left(t_{*}\right)\right) e^{-\gamma_{i j}\left(t_{*}\right) x_{i}^{*}\left(t_{*}-\tau_{i j}\left(t_{*}\right)\right)} \mid e^{\lambda t_{*}} \\
\leq & \lambda V_{i}\left(t_{*}\right)-\frac{a_{i i}\left(t_{*}\right) b_{i i}\left(t_{*}\right)}{\left(b_{i i}\left(t_{*}\right)+M\right)^{2}} V_{i}\left(t_{*}\right)+\sum_{j=1, j \neq i}^{n} \frac{a_{i j}\left(t_{*}\right) b_{i j}\left(t_{*}\right)}{\left(b_{i j}\left(t_{*}\right)+\kappa\right)^{2}} V_{i}\left(t_{*}\right) \\
& +\sum_{j=1}^{l} \beta_{i j}\left(t_{*}\right) \frac{1}{e^{2}} V_{i}\left(t_{*}-\tau_{i j}\left(t_{*}\right)\right) e^{\lambda \tau_{i j}\left(t_{*}\right)} \\
\leq & \left\{-\left[\frac{a_{i i}\left(t_{*}\right) b_{i i}\left(t_{*}\right)}{\left(b_{i i}\left(t_{*}\right)+M\right)^{2}}-\lambda\right]+\sum_{j=1, j \neq i}^{n} \frac{a_{i j}\left(t_{*}\right) b_{i j}\left(t_{*}\right)}{\left(b_{i j}\left(t_{*}\right)+\kappa\right)^{2}}+\sum_{j=1}^{l} \frac{\beta_{i j}\left(t_{*}\right)}{e^{2}} e^{\lambda r_{i}}\right\} K_{\varphi, x^{*} .}
\end{aligned}
$$

Thus,

$$
0 \leq-\left[\frac{a_{i i}\left(t_{*}\right) b_{i i}\left(t_{*}\right)}{\left(b_{i i}\left(t_{*}\right)+M\right)^{2}}-\lambda\right]+\sum_{j=1, j \neq i}^{n} \frac{a_{i j}\left(t_{*}\right) b_{i j}\left(t_{*}\right)}{\left(b_{i j}\left(t_{*}\right)+\kappa\right)^{2}}+\sum_{j=1}^{l} \frac{\beta_{i j}\left(t_{*}\right)}{e^{2}} e^{\lambda r_{i}},
$$

which contradicts (2.12). Hence (3.11) holds. It follows that

$$
\left|y_{i}(t)\right|<K_{\varphi, x^{*}} e^{\lambda t} \quad \text { for all } t>t_{\varphi, x^{*}}, i=1,2, \ldots, n .
$$

This completes the proof.

Remark 1. For the above system, Chen [3] have considered the permanence of it and Wang et al. [28] have investigated its exponential extinction. Chen and Wang et al. [5] have studied the existence and global exponential stability of positive almost periodic solutions for system (1.2) with nonlinear densitydependent mortality terms $D_{i j}(t, N)=a_{i j}(t)-b_{i j}(t) e^{-N}$. It is clear that our work complement the existence and global exponential stability of positive almost periodic solutions for system (1.2) with nonlinear density-dependent mortality terms $D_{i j}(t, N)=\frac{a_{i j}(t) N}{b_{i j}(t)+N}$.

\section{Acknowledgements}

The authors are grateful to the anonymous reviewers for his/her thoughtful comments and constructive suggestions on improving our original manuscript. This work is partially supported by the National Natural Science Foundation of China (No. 11601405) and the Fundamental Research Funds for the Central Universities (No. 2452015086, 2014YB023).

\section{References}

[1] H. Bereketoglu, G. Seyhan and A. Ogun. Advanced impulsive differential equations with piecewise constant arguments. Mathematical Modelling and Analysis, 15(2):175-187, 2011. https://doi.org/10.3846/1392-6292.2010.15.175-187. 
[2] L. Berezansky, E. Braverman and L. Idels. Nicholson's blowflies differential equations revisited: Main results and open problems. Applied Mathematical Modelling, 34(6):1405-1417, 2010. https://doi.org/10.1016/j.apm.2009.08.027.

[3] W. Chen. Permanence for Nicholson-type delay systems with patch structure and nonlinear density-dependent mortality terms. Electronic Journal of Qualitative Theory of Differential Equations, 73:1-14, 2012. https://doi.org/10.14232/ejqtde.2012.1.73.

[4] W. Chen and B.W. Liu. Positive almost periodic solution for a class of Nicholson's blowflies model with multiple time-varying delays. Journal of Computational and Applied Mathematics, 235(8):2090-2097, 2011. https://doi.org/10.1016/j.cam.2010.10.007.

[5] W. Chen and W.T. Wang. Almost periodic solutions for a delayed Nicholson's blowflies system with nonlinear density-dependent mortality terms and patch structure. Advances in Difference Equations, 2014(1):205, 2014. https://doi.org/10.1186/1687-1847-2014-205.

[6] F. Chérif. Analysis of global asymptotic stability and pseudo almost periodic solution of a class of chaotic neural networks. Mathematical Modelling and Analysis, 18(4):489-504, 2013. https://doi.org/10.3846/13926292.2013.840686.

[7] T. Faria. Global asymptotic behaviour for a Nicholson model with patch structure and multiple delays. Nonlinear Analysis: Theory, Methods $\&$ Applications, 74(18):7033-7046, 2011. https://doi.org/10.1016/j.na.2011.07.024.

[8] A.M. Fink. Almost Periodic Differential Equations. Springer, Berlin, Heidelberg, 1974. https://doi.org/10.1007/BFb0070324. Lecture Notes in Mathematics. Vol. 377

[9] W.S.C. Gurney, S.P. Blythe and R.M. Nisbet. Nicholson's blowflies revisited. Nature, 287(5777):17-21, 1980. https://doi.org/10.1038/287017a0.

[10] J.K. Hale and S.M. Verduyn Lunel. Introduction to Functional Differential Equations. Springer-Verlag, New York, 1993. https://doi.org/10.1007/978-1-46124342-7. Applied Mathematical Sciences. Vol. 99

[11] C.Y. He. Almost Periodic Differential Equations. Higher Education Publishing House, Beijing, 1992. (in Chinese)

[12] B. Liu and S. Gong. Permanence for Nicholson-type delay systems with nonlinear density-dependent mortality terms. Nonlinear Analysis: Real World Applications, 12(4):1931-1937, 2011. https://doi.org/10.1016/j.nonrwa.2010.12.009.

[13] B.W. Liu. Global stability of a class of Nicholson's blowflies model with patch structure and multiple time-varying delay. Nonlinear Analysis: Real World Applications, 11(4):2557-2562, 2010. https://doi.org/10.1016/j.nonrwa.2009.08.011.

[14] B.W. Liu. The existence and uniqueness of positive periodic solutions of Nicholson-type delay systems. Nonlinear Analysis: Real World Applications, 12(6):3145-3151, 2011. https://doi.org/10.1016/j.nonrwa.2011.05.014.

[15] B.W. Liu. Almost periodic solutions for a delayed Nicholson's blowflies model with a nonlinear density-dependent mortality term. Advances in Difference Equations, 2014(1):72, 2014. https://doi.org/10.1186/1687-1847-2014-72.

[16] X.G. Liu and J.X. Meng. The positive almost periodic solution for Nicholson-type delay systems with linear harvesting terms. Applied Mathematical Modelling, 36(7):3289-3298, 2012. https://doi.org/10.1016/j.apm.2011.09.087. 
[17] F. Long. Positive almost periodic solution for a class of Nicholson's blowflies model with a linear harvesting term. Nonlinear Analysis: Real World Applications, 13(2):686-693, 2012. https://doi.org/10.1016/j.nonrwa.2011.08.009.

[18] F. Long and B.W. Liu. Existence and uniqueness of positive periodic solutions of delayed Nicholson's blowflies models. Annales Polonici Mathematici, 103(3):217-228, 2012. https://doi.org/10.4064/ap103-3-1.

[19] H.L. Ma and H. Feng. Dynamic analysis for Bertrand competition model with exponential form. Mathematical Modelling and Analysis, 21(6):741-751, 2016. https://doi.org/10.3846/13926292.2016.1237388.

[20] A.J. Nicholson. An outline of the dynamics of animal populations. Australian Journal of Zoology, 2(1):9-65, 1954. https://doi.org/10.1071/ZO9540009.

[21] S.H. Saker and S. Agarwal. Oscillation and global attractivity in a periodic Nicholson's blowflies model. Mathematical and Computer Modelling, 35(7):719731, 2002. https://doi.org/10.1016/S0895-7177(02)00043-2.

[22] H. Smith. An Introduction to Delay Differential Equations with Applications to the Life Sciences. Springer, New York, 2011. https://doi.org/10.1007/978-14419-7646-8. Texts in Applied Mathematics. Vol. 57

[23] H.L. Smith. Monotone Dynamical Systems: An introductionnto the Theory of Competitive and Cooperative Systems. AMS, Providence RI, 1995. Mathematical Surveys and Monographs. Vol 41.

[24] X.-K. Sun, H.-F. Huo and X.-B. Zhang. A predator-prey model with functional response and stage structure for prey. Abstract and Applied Analysis, 2012:1-19, 2012. https://doi.org/10.1155/2012/628103.

[25] Y. Takeuchi, W.D. Wang and Y. Saito. Global stability of population models with patch structure. Nonlinear Analysis: Real World Applications, 7(2):235247, 2006. https://doi.org/10.1016/j.nonrwa.2005.02.005.

[26] C. Tian and P. Zhu. A plankton allelopathic model described by a delayed quasilinear parabolic system. Mathematical Modelling and Analysis, 17(4):485497, 2012. https://doi.org/10.3846/13926292.2012.706652.

[27] J. Tumwiine, J.Y.T. Mugisha and L.S. Luboobi. Threshold and stability results for a malaria model in a population with protective intervention among high-risk groups. Mathematical Modelling and Analysis, 13(3):443-460, 2008. https://doi.org/10.3846/1392-6292.2008.13.443-460.

[28] W. Wang. Exponential extinction of Nicholson's blowflies system with nonlinear density-dependent mortality terms. Abstract and Applied Analysis, 2012:1-15, 2012. https://doi.org/10.1155/2012/302065.

[29] W. Wang. Positive periodic solutions of delayed Nicholson's blowflies models with a nonlinear density-dependent mortality term. Applied Mathematical Modelling, 36(10):4708-4713, 2012. https://doi.org/10.1016/j.apm.2011.12.001.

[30] W. Wang, L. Wang and W. Chen. Existence and exponential stability of positive almost periodic solution for Nicholson-type delay systems. Nonlinear Analysis: Real World Applications, 12(4):1938-1949, 2011. https://doi.org/10.1016/j.nonrwa.2010.12.010.

[31] Y. Xu. Existence and global exponential stability of positive almost periodic solutions for a delayed Nicholson's blowflies model. Journal of the Korean Mathematical Society, 51(3), 2014. https://doi.org/10.4134/JKMS.2014.51.3.473. 
[32] X. Zhang, R. Xu and Z. Li. Global stability of a three-species food-chain model with diffusion and nonlocal delays. Mathematical Modelling and Analysis, 16(3):376-389, 2011. https://doi.org/10.3846/13926292.2011.601769.

[33] X.-B. Zhang, H.-F. Huo, H. Xiang and X.-Y. Meng. Dynamics of the deterministic and stochastic SIQS epidemic model with non-linear incidence. Applied Mathematics and Computation, 243:546-558, 2014. https://doi.org/10.1016/j.amc.2014.05.136. 